\title{
Nonlocal Fractional Hybrid Boundary Value Problems Involving Mixed Fractional Derivatives and Integrals via a Generalization of Darbo's Theorem
}

\author{
Ayub Samadi, ${ }^{1}$ Sotiris K. Ntouyas $\mathbb{D D}^{2},{ }^{2}$ and Jessada Tariboon ${ }^{3}{ }^{3}$ \\ ${ }^{1}$ Department of Mathematics, Miyaneh Branch, Islamic Azad University, Miyaneh, Iran \\ ${ }^{2}$ Department of Mathematics, University of Ioannina, Ioannina 451 10, Greece \\ ${ }^{3}$ Intelligent and Nonlinear Dynamic Innovations Research Center, Department of Mathematics, Faculty of Applied Science, \\ King Mongkut's University of Technology North Bangkok, Bangkok 10800, Thailand
}

Correspondence should be addressed to Jessada Tariboon; jessada.t@sci.kmutnb.ac.th

Received 6 December 2020; Revised 8 January 2021; Accepted 13 January 2021; Published 29 January 2021

Academic Editor: Ahmet Ocak Akdemir

Copyright (C) 2021 Ayub Samadi et al. This is an open access article distributed under the Creative Commons Attribution License, which permits unrestricted use, distribution, and reproduction in any medium, provided the original work is properly cited.

In this work, a new existence result is established for a nonlocal hybrid boundary value problem which contains one left Caputo and one right Riemann-Liouville fractional derivatives and integrals. The main result is proved by applying a new generalization of Darbo's theorem associated with measures of noncompactness. Finally, an example to justify the theoretical result is also presented.

\section{Introduction}

In the past years, fractional differential equations have attracted a lot of attention from many research studies as they have played a key role in many basic sciences such as chemistry, control theory, biology, and other arenas [1-3]. In addition, boundary conditions of differential models are the strongest tools to extend applications of those equations [4-6]. In fact, fractional differential equations can be extended by creating different types of boundary conditions. Newly, many authors have studied various types of boundary conditions to obtain new results of differential models.

The following hybrid differential equation was studied by Dhage and Lakshmikantham [7]:

$$
\left\{\begin{array}{l}
\frac{\mathrm{d}}{\mathrm{d} t}\left[\frac{x(t)}{h(t, x(t))}\right]=\omega(t, x(t)), \quad \text { a.e } t \in J, \\
x\left(t_{0}\right)=x_{0} \in \mathbb{R},
\end{array}\right.
$$

where $h$ and $\omega$ are continuous functions from $J \times \mathbb{R}$ into $\mathbb{R} \backslash\{0\}$ and $\mathbb{R}$, respectively. Based on the above work, the Caputo hybrid boundary value problem of the form

$$
\left\{\begin{array}{l}
{ }^{C} D_{0^{+}}^{p}\left[\frac{x(t)}{h(t, x(t))}\right]=\omega(t, x(t)), \quad \text { a.e } t \in I:=[0, L], \\
a_{1} \frac{x(0)}{h(0, x(0))}+a_{2} \frac{x(L)}{h(L, x(L))}=d,
\end{array}\right.
$$

was studied by Hilal and Kajouni [8] in which $0<p<1, h$ and $\omega$ are continuous functions from $J \times \mathbb{R}$ into $\mathbb{R} \backslash\{0\}$ and $\mathbb{R}$, respectively, and $a_{1}, a_{2}$, and $d$ are real constants with $a_{1}+a_{2} \neq 0$. For some recent results on hybrid fractional differential equations, see [9-12].

In [13], the authors proved the following integro-differential equation: 


$$
\begin{aligned}
{ }^{c} D_{1_{-}}^{\alpha_{1} \mathrm{RL}} D_{0_{+}}^{\alpha_{2}} u(t)+\theta I_{-1}^{p} I_{0_{+}}^{q} f_{1}(t, u(t)) & =f(t, u(t)), \quad t \in[0,1], \\
u(0) & =u(\xi)=0, \\
u(1) & =\delta u(\mu), \quad 0<\xi<\mu<1,
\end{aligned}
$$

where ${ }^{c} D_{1}^{\alpha_{1}}$ and ${ }^{\mathrm{RL}} D_{0_{+}}^{\alpha_{2}}$ indicate right Caputo and left Riemann-Liouville fractional derivatives of orders $\alpha_{1} \in(1,2]$ and $\alpha_{2} \in(0,1], \quad$ respectively, $f_{1}, f_{2}:[0,1] \times \mathbb{R} \longrightarrow \mathbb{R}$ are continuous functions, and the symbols $I_{-1}^{p}$ and $I_{0^{+}}^{q}$ denote both right and left Riemann-Liouville fractional integrals of orders $p, q>0$, respectively. Ahmad et al. [13] applied Banach and Krasnosel'skiǐ fixed point theorems as well
Leray-Schauder nonlinear alternative to obtain main results. We point out that fractional differential equations containing mixed fractional derivatives appear in the study of variational principles [14].

For some recent results for boundary value problems involving left or/and right fractional derivatives, we refer to the papers [15-31] and references therein.

In the present paper, we combine mixed fractional derivatives and hybrid fractional differential equations. More precisely, we investigate the existence of solutions for the following hybrid boundary value problem which contains both left Caputo and right Riemann-Liouville fractional derivatives and integrals and nonlocal hybrid conditions of the form:

$$
\begin{aligned}
{ }^{c} D_{1_{-}}^{\alpha_{1} \mathrm{RL}} D_{1_{+}}^{\alpha_{2}} \frac{u(t)}{g(t, u(t))}+\theta I_{-1}^{p} I_{0^{+}}^{q} f_{1}(t, u(t)) & =f_{2}(t, y(t)), \quad t \in J:=[0,1], \\
\frac{\frac{u(0)}{g(0, u(0))}}{g(\xi, u(\xi))}=0, & \frac{u(\xi)}{\frac{u(1)}{g(1, u(1))}}=\delta \frac{u(\mu)}{g(\mu, u(\mu))}, \quad 0<\xi<\mu<1,
\end{aligned}
$$

where ${ }^{c} D_{1_{-}}^{\alpha_{1}}$ and ${ }^{\mathrm{RL}} D_{0_{+}}^{\alpha_{2}}$ are right Caputo and left Riemann-Liouville fractional derivatives of orders $\alpha_{1} \in(1,2]$ and $\alpha_{2} \in(0,1]$, respectively, and the symbols $I_{-1}^{p}$ and $I_{0^{+}}^{q}$ denote both right and left Riemann-Liouville fractional integrals of orders $p, q>0, \quad$ respectively, $f_{1}, f_{2} \in C(J \times \mathbb{R}, \mathbb{R}), g \in C(J \times \mathbb{R}, \mathbb{R} \backslash\{0\})$, and $\delta, \theta \in \mathbb{R}$. An existence result is obtained via a new extension of Darbo's theorem associated with measures of noncompactness.

The structure of the paper has been organized as follows. Section 2 presents some basic definitions and lemmas which will be applied in the future. In Section 3, we prove an existence result for problem (4). Finally, we present an example to illustrate the obtained result.

\section{Preliminaries}

Now, some basic notations are recalled from [2].

Definition 1. For an integrable function $\phi:(0, \infty) \longrightarrow \mathbb{R}$, we define the left and right Riemann-Liouville fractional integrals of order $\beta>0$, respectively, by

$$
\begin{aligned}
I_{0^{+}}^{\beta} \phi(t)= & \int_{0}^{t} \frac{(t-s)^{\beta-1}}{\Gamma(\beta)} \phi(s) \mathrm{d} s, \\
& I_{1-}^{\beta} \phi(t) \int_{t}^{1} \frac{(s-t)^{\beta-1}}{\Gamma(\beta)} \phi(s) \mathrm{d} s .
\end{aligned}
$$

Definition 2. For the function $\phi:(0, \infty) \longrightarrow \mathbb{R}$ in which $\phi \in C^{n}(0, \infty)$, we define the left Riemann-Liouville fractional derivative and the right Caputo fractional derivative of order $\beta \in(n-1, n]$, respectively, by

$$
\begin{aligned}
{ }^{\mathrm{RL}} D_{0^{+}}^{\beta} \phi(t) & =\frac{\mathrm{d}^{n}}{\mathrm{~d} t^{n}} \int_{0}^{t} \frac{(t-s)^{n-\beta-1}}{\Gamma(n-\beta)} \phi(s) \mathrm{d} s, \\
{ }^{c} D_{1_{-}}^{\beta} \phi(t) & =(-1)^{n} \int_{t}^{1} \frac{(s-t)^{n-\beta-1}}{\Gamma(n-\beta)} \phi^{(n)}(s) \mathrm{d} s .
\end{aligned}
$$

Lemma 1. If $p>0$ and $q>0$, then the following relations hold almost everywhere on $[a, b]$ :

$$
\begin{aligned}
& I_{1-}^{p} I_{-1}^{q} f(x)=I_{1-}^{p+q} f(x), \\
& I_{0^{+}}^{p} I_{0^{+}}^{q} f(x)=I_{0^{+}}^{p+q} f(x) .
\end{aligned}
$$

As the technique of measure of noncompactness will be applied to obtain our main result, we recall some basic facts about the notion of measure of noncompactness.

Assume that $Z$ is the real Banach space with the norm $\|\cdot\|$ and zero element $\theta$. For a nonempty subset $X$ of $Z$, the closure and the closed convex hull of $X$ will be denoted by $\bar{X}$ and Conv $(X)$, respectively. Also, $M_{Z}$ and $N_{Z}$ denote the family of all nonempty and bounded subsets of $Z$ and its subfamily consisting of all relatively compact sets, respectively.

Definition 3 (see [32]). We say that a mapping $h: M_{Z} \longrightarrow[0, \infty)$ is a measure of noncompactness, if the following conditions hold true: 
(1) The family Kerh $=\left\{X \in M_{Z}: h(X)=0\right\}$ is nonempty and $\operatorname{Ker} h \subseteq N_{Z}$

(2) $X_{1} \subseteq Y_{1} \Rightarrow h\left(X_{1}\right) \leq h\left(Y_{1}\right)$

(3) $h(\bar{X})=h(X)$

(4) $h(\operatorname{Conv}(X))=h(X)$

(5) $h(\alpha X+(1-\alpha) Y) \leq \alpha h(X)+(1-\alpha) h(Y)$ for $\alpha \in[0,1]$

(6) For the sequence $\left\{X_{n}\right\}$ of closed sets from $M_{Z}$ in which $X_{n+1} \subseteq X_{n}$ for $n=1,2, \ldots$ and $\lim _{n \longrightarrow \infty} h\left(X_{n}\right)=0$, we have $\cap_{n=1}^{\infty} X_{n} \neq \varnothing$

In [33], some generalizations of Darbo's theorem have been proved by Samadi and Ghaemi. Also, in [34], Darbo's theorem was extended, and the following result was presented which is basis for our main result.

Theorem 1. Let $T$ be a continuous self-mapping operator on the set $D$, where $D$ denotes a nonempty, bounded, closed, and convex subset of a Banach space Z. Assume that, for all nonempty subset $X$ of $D$, we have

$$
\theta_{1}\left((h(X))+\theta_{2}(h(T(X))) \leq \theta_{2}(h(X))\right),
$$

where $h$ is an arbitrary measure of noncompactness defined in $Z$ and $\left(\theta_{1}, \theta_{2}\right) \in U$. Then, $T$ has a fixed point in $D$.

In Theorem 1 , let $U$ indicate the set of all pairs $\left(\theta_{1}, \theta_{2}\right)$ where the following conditions hold true:

$\left(U_{1}\right) \theta_{1}\left(t_{n}\right) \nrightarrow 0$ for each strictly increasing sequence $\left\{t_{n}\right\}$

$\left(U_{2}\right) \theta_{2}$ is strictly increasing function
$\left(U_{3}\right)$ If $\left\{\alpha_{n}\right\}$ be a sequence of positive numbers, then $\lim _{n \longrightarrow \infty} \alpha_{n}=0 \Leftrightarrow \lim _{n \longrightarrow \infty} \theta_{2}\left(\alpha_{n}\right)=-\infty$

$\left(U_{4}\right)$ Let $\left\{l_{n}\right\}$ be a decreasing sequence in which $l_{n} \longrightarrow 0$ and $\theta_{1}\left(l_{n}\right)<\theta_{2}\left(l_{n}\right)-\theta_{2}\left(l_{n+1}\right)$, then we have $\sum_{n=1}^{\infty} l_{n}<\infty$

Next, the definition of a measure of noncompactness in the space $C([0,1])$ is recalled which will be applied later. Fix $Y \in M_{C[0,1]}$, and for $\varepsilon>0$ and $y \in Y$, we define

$$
\begin{aligned}
\varphi(y, \varepsilon) & =\sup \{|y(t)-y(s)|: t, s \in[0,1], \quad|t-s| \leq \varepsilon\}, \\
\varphi(Y, \varepsilon) & =\sup \{\varphi(y, \varepsilon): y \in Y\}, \\
\varphi_{0}(Y) & =\lim _{\varepsilon \longrightarrow 0} \varphi(Y, \varepsilon) .
\end{aligned}
$$

Banas and Goebel [32] proved that $\varphi_{0}(Y)$ is a measure of noncompactness in the space $C([0,1])$.

Lemma 2 (see [32]). The measure of noncompactness $\varphi_{0}$ on $C(I)$ satisfies the following condition:

$$
\varphi_{0}(X Y) \leq\|X\| \varphi_{0}(Y)+\|Y\| \varphi_{0}(X),
$$

for all $X, Y \subseteq C(I)$.

\section{Main Existence Result}

In this section, an existence result of problem (4) is investigated. In view of [13], Lemma 2, we present the following lemma which is an essential tool in our consideration.

Lemma 3. Let $H_{1}, F_{1} \in C[0,1] \cap L(0,1), \quad g \in C([0,1]$, $\mathbb{R} \backslash\{0\})$, and $\Lambda \neq 0$. Then, the solution of the problem

$$
\left\{\begin{array}{l}
{ }^{c} D_{1_{-}}^{\alpha_{1} \mathrm{RL}} D_{0^{+}}^{\beta} \frac{u(t)}{g(t, u(t))}+\lambda I_{-1}^{p} I_{0_{+}}^{q} H_{1}(t)=F_{1}(t), \quad t \in[0,1], \\
\frac{u(0)}{g(0, u(0))}=\frac{u(\xi)}{g(\xi, u(\xi))}=0, \\
\frac{u(1)}{g(1, u(1))}=\delta \frac{u(\mu)}{g(\mu, u(\mu))}, \quad 0<\xi<\mu<1,
\end{array}\right.
$$

has the form:

$$
u(t)=g(t, u(t))\left[\int_{0}^{t} \frac{(t-s)^{\alpha_{2}-1}}{\Gamma\left(\alpha_{2}\right)}\left(I_{1_{-}}^{\alpha} F_{1}(s)-\lambda I_{1_{-}}^{\alpha_{1}+p} I_{0_{+}}^{q} H_{1}(s)\right) \mathrm{d} s+a_{1}(t)\left\{\begin{array}{c}
\delta \int_{0}^{\mu} \frac{(\mu-s)^{\alpha_{2}-1}}{\Gamma\left(\alpha_{2}\right)}\left(I_{1_{-}}^{\alpha} F_{1}(s)-\lambda I_{1_{-}}^{\alpha_{1}+p} I_{0_{+}}^{q} H(s)\right) \mathrm{d} s \\
-\int_{0}^{1} \frac{(1-s)^{\alpha_{2}-1}}{\Gamma\left(\alpha_{2}\right)}\left(I_{1_{-}}^{\alpha_{1}} F_{1}(s)-\lambda I_{1_{-}}^{\alpha_{1}+p} I_{0_{+}}^{q} H_{1}(s)\right) \mathrm{d} s
\end{array}\right\},\right.
$$


where

$$
\begin{aligned}
a_{1}(t) & =\frac{1}{\Lambda}\left[\xi^{\alpha_{2}+1} t^{\alpha_{2}}-\xi^{\alpha_{2}} t^{\alpha_{2}+1}\right], \\
a_{2}(t) & =\frac{1}{\Lambda}\left[t^{\alpha_{2}}\left(1-\delta \mu^{\alpha_{2}+1}\right)-t^{\alpha_{2}+1}\left(1-\delta \mu^{\alpha_{2}}\right)\right], \\
\Lambda & =\xi^{\alpha_{2}+1}\left(1-\delta \mu^{\alpha_{2}}\right)-\xi^{\alpha_{2}}\left(1-\delta \mu^{\alpha_{2}+1}\right) .
\end{aligned}
$$

Now, the hypotheses which will be applied to prove the main result of this section are presented.

$\left(H_{1}\right) g:[0,1] \times \mathbb{R} \longrightarrow \mathbb{R} \backslash\{0\}$ is a continuous function, and there exists a positive real number $d>0$ provided that

$$
\left|g\left(t, x_{1}\right)-g\left(t, x_{2}\right)\right| \leq e^{-d}\left|x_{1}-x_{2}\right|
$$

where $t \in I$ and $x_{1}, x_{2} \in \mathbb{R}$. Moreover, assume that $\bar{g}=\sup \{|g(t, 0)| ; t \in[0,1]\}$.

$\left(H_{2}\right) \quad f_{1}, f_{2}:[0,1] \times \mathbb{R} \longrightarrow \mathbb{R}$ are continuous functions provided that

$$
\begin{aligned}
\left|f_{1}(t, u)\right| & \leq M_{1}, \\
\left|f_{1}(t, u)-f_{1}(t, v)\right| & \leq k_{1}|u-v|, \\
\left|f_{2}(t, u)\right| & \leq M_{2}, \\
\left|f_{2}(t, u)-f_{2}(t, v)\right| & \leq k_{2}|u-v|,
\end{aligned}
$$

where $M_{1}, M_{2}, k_{1}, k_{2} \geq 0$ and $u, v \in \mathbb{R}$.

$\left(H_{3}\right)$ The inequality

$$
\left[e^{-d} r_{0}+\bar{g}\right]\left\{\frac{M_{1}}{\Gamma\left(\alpha_{1}+1\right)}+\frac{|\theta| M_{2}}{\Gamma\left(\alpha_{1}+p+1\right) \Gamma(q+1)}\right\} \Delta \leq r_{0}
$$

has a positive solution $r_{0}$. Also, assume that

$$
\left\{\frac{M_{1}}{\Gamma\left(\alpha_{1}+1\right)}+\frac{|\theta| M_{2}}{\Gamma\left(\alpha_{1}+p+1\right) \Gamma(q+1)}\right\} \Delta<1,
$$

where

$$
\begin{aligned}
& \Delta=\frac{1}{\Gamma\left(\alpha_{2}+1\right)}\left[1+\overline{a_{1}}\left(|\delta| \mu^{\alpha_{2}}+1\right)+\overline{a_{2}} \xi^{\alpha_{2}}\right], \\
& \overline{a_{1}}=\max _{t \in[0,1]}\left|a_{1}(t)\right|, \\
& \overline{a_{2}}=\max _{t \in[0,1]}\left|a_{2}(t)\right| .
\end{aligned}
$$

Theorem 2. Suppose that the hypotheses $\left(H_{1}\right)-\left(H_{3}\right)$ are true. Then, the hybrid boundary value problem (4) has at least one solution on $[0,1]$.

Proof. Due to Lemma 3, assume that the operator $T$ has been defined on $C(I), I:=[0,1]$ as follows:

$$
T_{1}(u)(t)=\left(F_{1} u(t)+\overline{F_{1}} u(t)\right)\left(G_{1} u(t)\right),
$$

where

$$
\begin{aligned}
G_{1} u(t)= & g(t, u(t)), \\
F_{1} u(t)= & \int_{0}^{t} \frac{(t-s)^{\alpha_{2}-1}}{\Gamma\left(\alpha_{2}\right)}\left(I_{1_{-}}^{\alpha_{1}} f_{2}(s, u(s))-\theta I_{1_{-}}^{\alpha_{1}+p} I_{0_{+}}^{q} f_{1}(s, u(s))\right) \mathrm{d} s, \\
\overline{F_{1}} u(t)= & a_{1}(t)\left\{\begin{array}{l}
\delta \int_{0}^{\mu} \frac{(\mu-s)^{\alpha_{1}-1}}{\Gamma\left(\alpha_{2}\right)}\left(I_{1_{-}}^{\alpha_{1}} f_{2}(s, u(s))-\theta I_{1_{-}}^{\alpha_{1}+p} I_{0_{+}}^{q} f_{2}(s, u(s))\right) \mathrm{d} s \\
-\int_{0}^{1} \frac{(1-s)^{\alpha_{2}-1}}{\Gamma\left(\alpha_{2}\right)}\left(I_{1_{-}}^{\alpha_{1}} f_{2}(s, u(s))-\theta I_{1_{-}}^{\alpha_{1}+p} I_{0_{+}}^{q} f_{1}(s, u(s))\right) \mathrm{d} s
\end{array}\right\} \\
& +a_{2}(t) \int_{0}^{\xi} \frac{(\xi-s)^{\alpha_{2}-1}}{\Gamma\left(\alpha_{2}\right)}\left(I_{1_{-}}^{\alpha_{1}} f_{2}(s, u(s))-\theta I_{1_{-}}^{\alpha_{1}+p} I_{0_{+}}^{q} f_{1}(s, u(s))\right) \mathrm{d} s .
\end{aligned}
$$

First, we show that $T_{1} u \in C(I)$ in which $u \in C(I)$. In view of assumption $\left(\mathrm{H}_{1}\right)$, we conclude that $G_{1} u \in C(I)$, $u \in C(I)$. Consequently, by proving $F_{1} u, \overline{F_{2}} u \in C(I)$, the claim is obtained. Let $l_{n}$ be a sequence in $[0,1]$ such that $l_{n} \longrightarrow l$. Then, due to our assumptions, we get 


$$
\begin{aligned}
\left|F_{1} u\left(l_{n}\right)-F_{1} u(l)\right| \leq & \left|\begin{array}{c}
\int_{0}^{l} \frac{\left[\left(l_{n}-s\right)^{\alpha_{2}-1}-(l-s)^{\alpha_{2}-1}\right]}{\Gamma\left(\alpha_{2}\right)}\left(I_{1_{-}}^{\alpha_{1}} f_{2}(s, u(s))-\theta I_{1_{-}}^{\alpha_{1}+p} I_{0_{+}}^{q} f_{1}(s, u(s))\right) \mathrm{d} s \mid \\
\quad+\int_{l}^{l_{n}} \frac{\left(l_{n}-s\right)^{\alpha_{2}-1}}{\Gamma\left(\alpha_{2}\right)}\left(I_{1_{-}}^{\alpha_{1}} f_{2}(s, u(s))-\theta I_{1_{-}}^{\alpha_{1}+p} I_{0_{+}}^{q} f_{1}(s, u(s))\right) \mathrm{d} s
\end{array}\right| \\
\leq & \frac{M_{1}}{\Gamma\left(\alpha_{2}\right)}\left|\int_{0}^{l_{n}}\left[\left(l_{n}-s\right)^{\alpha_{2}-1}-(l-s)^{\alpha_{2}-1}\right] I_{1-}^{\alpha_{1}}(1) \mathrm{d} s\right| \\
& +M_{1}\left|\frac{1}{\Gamma\left(\alpha_{2}\right)} \int_{l}^{l_{n}}\left(l_{n}-s\right)^{\alpha_{2}-1} I_{1_{-}}^{\alpha_{1}}(1) \mathrm{d} s\right|+\frac{|\theta| M_{2}}{\Gamma\left(\alpha_{2}\right)}\left|\int_{0}^{l}\left[\left(l_{n}-s\right)^{\alpha_{2}-1}-(l-s)^{\alpha_{2}-1}\right] I_{1_{-}}^{\alpha_{1}+p} I_{0_{+}}^{q}(1) \mathrm{d} s\right| \\
& +\frac{|\theta| M_{2}\left|\int_{l}\right| \int_{n}}{\Gamma\left(\alpha_{2}\right)}\left(l_{n}-s\right)^{\alpha_{2}-1} I_{1_{-}}^{\alpha_{1}+p} I_{0_{+}}^{q}(1) \mathrm{d} s \mid \leq\left\{\frac{M_{1}}{\Gamma\left(\alpha_{1}+1\right) \Gamma\left(\alpha_{2}+1\right)}+\frac{|\theta| M_{2}}{\Gamma(q+1) \Gamma\left(\alpha_{2}+1\right) \gamma\left(\alpha_{1}+p+1\right)}\right\} \\
& \times\left[2\left(l_{n}-l\right)^{\alpha_{2}}+\left|l_{n}^{\alpha_{2}}-l^{\alpha_{2}}\right|\right] \longrightarrow 0 .
\end{aligned}
$$

Hence, $F_{1} u \in C(I)$. To obtain that $\overline{F_{1}} u \in C(I)$, by the definitions of $a_{1}$ and $a_{2}$, we have $\left|a_{1}\left(l_{n}\right)-a_{1}(l)\right| \longrightarrow 0$ and $\left|a_{2}\left(l_{n}\right)-a_{2}(l)\right| \longrightarrow 0$. Hence, we have $\left|\overline{F_{1}} u\left(l_{n}\right)-\overline{F_{1}} u(l)\right| \longrightarrow 0$. Consequently, $T_{1} u \in C(I)$ for all $x \in C(I)$.
Now, we prove that the ball $D_{r_{0}}=\left\{u \in C(I):\|u\| \leq r_{0}\right\}$ is mapped into itself by the operator $T$. Let us fix $u \in C(I)$. Hence, due to existence assumptions, for $t \in I$, we have

$$
\left|\left(T_{1} u\right)(t)\right| \leq\left[e^{-d}|u(t)|+\bar{g}\right]
$$

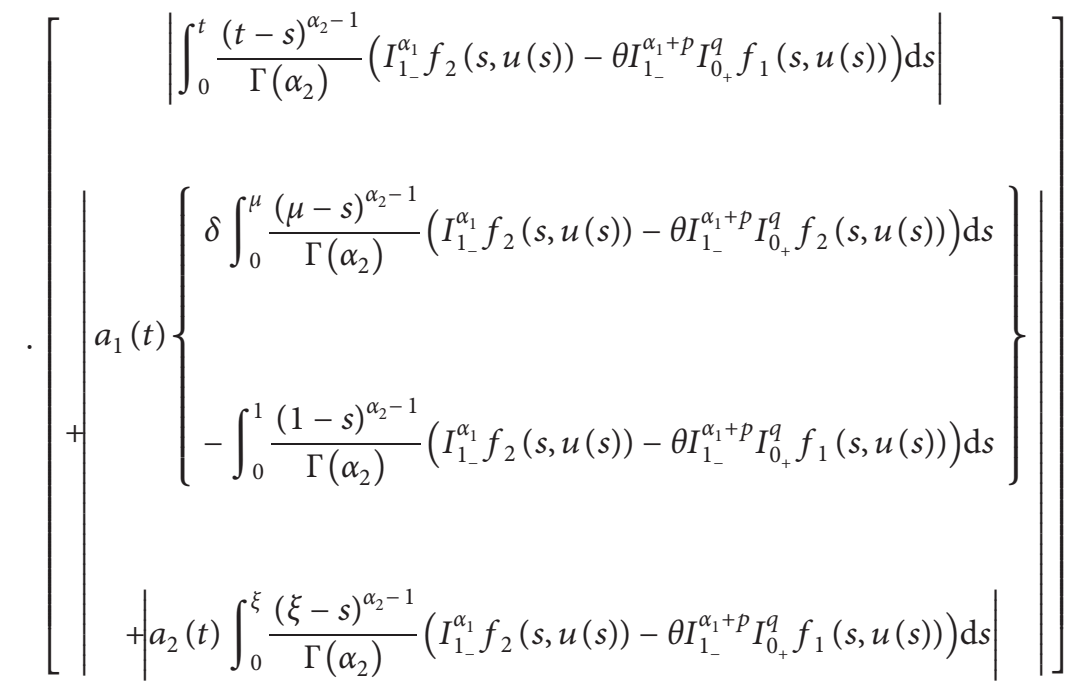

$$
\begin{aligned}
& \leq\left[e^{-d} r_{0}+\bar{g}\right]\left\{\frac{M_{1}}{\Gamma\left(\alpha_{1}+1\right)}+\frac{|\theta| M_{2}}{\Gamma\left(\alpha_{1}+p+1\right) \Gamma(q+1)}\right\} \Delta .
\end{aligned}
$$

Consequently, according to assumption $\left(\mathrm{H}_{3}\right)$ we conclude that $T$ maps the ball $D_{r_{0}}$ into itself.
Now, the continuity property of the operator $T$ is considered on the ball $D_{r_{0}}$. To do this, fix $\varepsilon>0$ and take $u, v \in D_{r_{0}}$ such that $\|u-v\| \leq \varepsilon$. Then, for $t \in I$, we have 


$$
\begin{aligned}
\left|\left(T_{1} u\right)(t)-\left(T_{1} v\right)(t)\right|= & \left|F_{1} u(t) G_{1} u(t)-F_{1} v(t) G_{1} v(t)+\overline{F_{1}} u(t) G_{1} u(t)-\overline{F_{1}} v(t) G_{1} v(t)\right| \\
\leq & \left|F_{1} u(t) G_{1} u(t)-F_{1} v(t) G_{1} u(t)\right|+\left|F_{1} v(t) G_{1} u(t)-F_{1} v(t) G_{1} v(t)\right| \\
& +\left|\overline{F_{1}} u(t) G_{1} u(t)-\overline{F_{1}} v(t) G_{1} u(t)\right|+\left|\overline{F_{1}} v(t) G_{1} u(t)-\overline{F_{1}} v(t) G_{1} v(t)\right| \\
\leq & |g(t, u(t))-g(t, v(t))|\left|F_{1} v(t) \|+\right| g(t, u(t))\left|F_{1} u(t)-F_{1} v(t)\right| \\
& +|g(t, u(t))-g(t, v(t))|\left|\overline{F_{1}} v(t) \|+\right| g(t, u(t))\left|\overline{F_{1}} u(t)-\overline{F_{1}} v(t)\right| .
\end{aligned}
$$

Then, we have

$$
\begin{aligned}
& \left|\left(T_{1} u\right)(t)-\left(T_{1} v\right)(t)\right| \leq e^{-d} \varepsilon\left(F_{1} v(t)+\overline{F_{1}} v(t)\right)+|g(t, u(t))| \varepsilon\left[\int_{0}^{t} \frac{(t-s)^{\alpha_{2}-1}}{\Gamma\left(\alpha_{2}\right)}\left(k_{1} I_{1_{-}}^{\alpha_{1}}(1)+k_{2}|\theta| I_{1_{-}}^{\alpha_{1}+p} I_{0_{+}}^{q}(1)\right) \mathrm{d} s\right]
\end{aligned}
$$

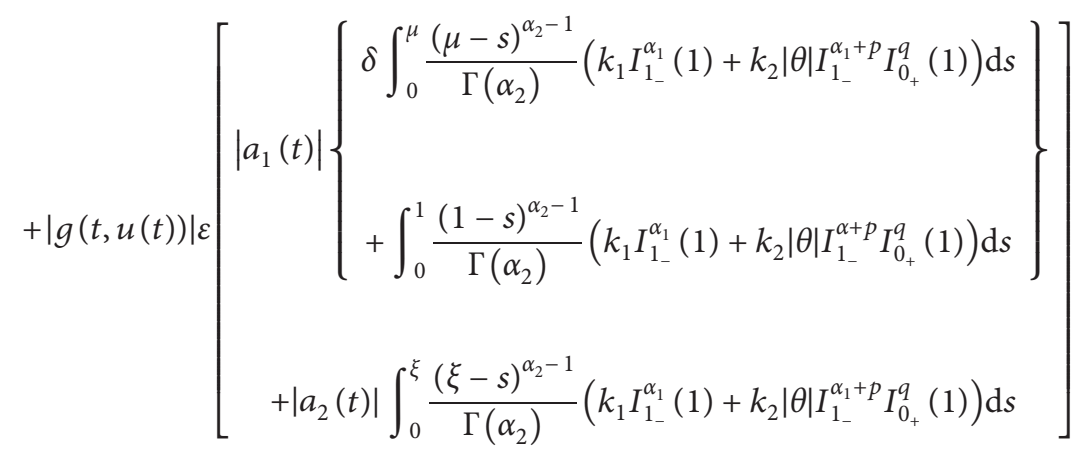

$$
\begin{aligned}
& \leq \varepsilon\left[\frac{k_{1}}{\Gamma\left(\alpha_{1}+1\right)}+\frac{|\theta| k_{2}}{\Gamma\left(\alpha_{1}+p+1\right) \Gamma(q+1)}\right]\left\{e^{-d} \Delta+\left(e^{-d} r_{0}+\bar{g}\right)(\Delta+1)\right\}
\end{aligned}
$$

Consequently, the continuity property of $T$ is obtained on the ball $D_{r_{0}}$.

To finish the proof, condition (8) of Theorem 1 is proved. Consider $X$ as a nonempty subset of the ball $D_{r_{0}}$ and assume that $u \in X, \varepsilon>0$ be arbitrarily constant. Choose $l_{1}, l_{2} \in[0,1]$ such that $l_{1}<l_{2}$ and $\left|l_{2}-l_{1}\right|<\varepsilon$. Taking into account our assumptions, we get

$$
\begin{aligned}
\left(\left|\left(G_{1} u\right)\left(l_{1}\right)-\left(G_{1} u\right)\left(l_{2}\right)\right|\right)= & \left|g\left(l_{1}, u\left(l_{1}\right)\right)-g\left(l_{2}\right), u\left(l_{2}\right)\right| \\
\leq & \left|g\left(l_{1}, u\left(l_{1}\right)\right)-g\left(l_{1}\right), u\left(l_{2}\right)\right| \\
& +\left|g\left(l_{1}, u\left(l_{2}\right)\right)-g\left(l_{2}\right), u\left(l_{2}\right)\right| \\
\leq & e^{-d} \varphi(X, \varepsilon)+\varphi(g, \varepsilon),
\end{aligned}
$$

where

$$
\begin{aligned}
\varphi(g, \varepsilon) & =\sup \left\{\left|g\left(l_{1}, u\right)-g\left(l_{2}, u\right)\right| ; l_{1}, l_{2} \in I,\left|l_{1}-l_{2}\right|\right. \\
& \left.<\varepsilon, u \in\left[-r_{0}, r_{0}\right]\right\} .
\end{aligned}
$$

Consequently,

$$
\varphi\left(G_{1} X, \varepsilon\right) \leq e^{-d} \varphi(X, \varepsilon)+\varphi(g, \varepsilon) .
$$

As $g$ is uniformly continuous on $I \times\left[-r_{0}, r_{0}\right]$, we have $\varphi(g, \varepsilon) \longrightarrow 0$ as $\varepsilon \longrightarrow 0$. Thus, from (27), we conclude that

$$
\varphi_{0}\left(G_{1} X\right) \leq e^{-d} \varphi_{0}(X)
$$

Next, we estimate $\varphi_{0}\left(F_{1} X\right)$ and $\varphi_{0}\left(\overline{F_{1}}(X)\right)$. In view of (21), since $F_{1} x$ is uniformly continuous on $[0,1]$, then for fixed $\varepsilon>0$, there exists $\delta>0$ such that, for $l_{1}, l_{2} \in I$ with $\left|l_{2}-1_{1}\right|<\delta \leq \varepsilon$, we have

$$
\varphi_{0}\left(F_{1} X\right) \leq \mathcal{\varepsilon}
$$

Besides, since $a_{1}$ and $a_{2}$ are uniformly continuous on $[0,1]$, for $l_{1}, l_{2} \in[0,1]$ with $\left|l_{2}-1_{1}\right|<\delta \leq \varepsilon$, we have $\left|a_{2}\left(l_{2}\right)-a_{2}\left(l_{1}\right)\right|<\varepsilon$ and also $\left|a_{1}\left(l_{2}\right)-a_{1}\left(l_{1}\right)\right|<\varepsilon$. Consequently, we conclude that $\varphi_{0}\left(\overline{F_{1}}(X)\right)=0$. Now, we estimate $\varphi_{0}\left(T_{1} X\right)$ for $X \subseteq D_{r_{0}}$. By applying (28) and (29) and Lemma 2 and using the fact that $\varphi_{0}\left(\overline{F_{1}}(X)\right)=0$, we get 


$$
\begin{aligned}
\varphi_{0}\left(T_{1} X\right)= & \varphi_{0}\left(G_{1}\right) X\left(F_{1}\right)(X)+\overline{F_{1}}((X)) \\
\leq & \left(\left\|F_{1} X\right\|+\left\|\overline{F_{1}}(X)\right\|\right) \varphi_{0}\left(G_{1} X\right) \\
& +\left\|G_{1} X\right\|\left(\varphi_{0}\right)\left(F_{1} X\right)+\varphi_{0}\left(\overline{F_{1}}\right)((X)) \\
\leq & e^{-d} \varphi_{0}(X)\left\{\frac{M_{1}}{\Gamma\left(\alpha_{1}+1\right)}+\frac{|\theta| M_{2}}{\Gamma\left(\alpha_{1}+p+1\right) \Gamma(q+1)}\right\} \Delta .
\end{aligned}
$$

Consequently, we derive that

$$
d+\ln \left(\varphi_{0}\left(T_{1} X\right)\right) \leq \ln \left(\varphi_{0}(X)\right) .
$$

Thus, we conclude the contractive condition in Theorem 1 with $\theta_{1}(t)=d$ and $\theta_{2}(t)=\ln (t)$. Thus, by Theorem 1 , at least one solution is obtained for the operator $T$ in $D_{r_{0}}$ which is a solution of problem (4) and the proof is completed.

Now, the following example is investigated to show the applicability of the obtained result.

Example 1. Consider the following hybrid boundary value problem:

$$
\begin{aligned}
& D_{1-}^{(3 / 2)} D_{0^{+}}^{(1 / 2)} \frac{u(t)}{\left(e^{-d} / 1+t+|u(t)|\right)}+2 I_{1-}^{4 / 3} I_{0^{+}}^{5 / 4} \frac{e^{-t}}{100} \cos u(t)=\frac{e^{-t}}{100} \sin u(t), \\
& \begin{aligned}
\frac{u(0)}{\left(e^{-d} / 1+|u(0)|\right)}=\frac{u(2 / 3)}{\left(e^{-d} / t+1+|u(2 / 3)|\right)}=0, \frac{u(1)}{\left(e^{-d} / 2+|u(1)|\right)}=\frac{1}{2} \frac{u(3 / 4)}{\left(e^{-d} / 1+(3 / 4)+|u(3 / 4)|\right)}=0 . \\
\text { By putting }
\end{aligned} \quad\left[e^{-d} r_{0}+\frac{1}{100}\right]\left\{\frac{(1 / 100)}{\Gamma(5 / 2)}+\frac{(2 / 100)}{\Gamma(23 / 6) \Gamma(9 / 4)}\right\} \Delta \leq r_{0} .
\end{aligned}
$$

$$
\begin{aligned}
g(t, u(t)) & =\frac{e^{-d}}{1+t+|u(t)|}, \\
f_{1}(t, u(t)) & =\frac{e^{-t}}{100} \cos u(t), \\
f_{2}(t, u(t)) & =\frac{e^{-t}}{100} \sin u(t),
\end{aligned}
$$

in problem (4), we conclude the above hybrid boundary value problem as a special case of problem (4). Now, the conditions of Theorem 2 are checked. For all $l \in[0,1]$ and $u_{1}, u_{2} \in \mathbb{R}$, we have

$$
\left|g\left(l, u_{1}\right)-g\left(l, u_{2}\right)\right| \leq e^{-d}\left|u_{1}-u_{2}\right| .
$$

Moreover, we have $\bar{g}=\sup \{\mid g(l, 0) ; l \in[0,1]\}=e^{-d}$. Besides, the functions $f_{1}$ and $f_{2}$ are continuous, and for all $l \in[0,1]$ and $u, v \in \mathbb{R}$, we have

$$
\begin{gathered}
\left|f_{2}(t, u)\right| \leq \frac{1}{100}, \\
\left|f_{2}(t, u)-f_{2}(t, v)\right| \leq \frac{1}{100}|u-v|, \\
\left|f_{1}(t, u)\right| \leq \frac{1}{100}, \\
\left|f_{1}(t, u)-f_{1}(t, v)\right| \leq \frac{1}{100}|u-v| .
\end{gathered}
$$

In this example $\alpha_{1}=3 / 2, \alpha_{2}=1 / 2, \theta=2, p=4 / 3$, $q=5 / 4, \mu=3 / 43 / 4, \delta=1 / 2$, and $\xi=2 / 3$. Hence, $|\Lambda| \approx 0.242$, $\overline{a_{1}}=1.121$ and $\overline{a_{2}}=1.168$. Consequently, the existent inequality in condition $\left(\mathrm{H}_{3}\right)$ has the form:

Obviously, the above inequality has the positive solution $r_{0}$, for example $r_{0}=1$. Moreover, according to the obtained values we have

$$
\left\{\frac{M_{1}}{\Gamma\left(\alpha_{1}+1\right)}+\frac{|\theta| M_{2}}{\Gamma\left(\alpha_{1}+p+1\right) \Gamma(q+1)}\right\} \Delta<1 .
$$

Thus, we conclude all conditions of Theorem 2, and hence at least one solution of the mapping $T_{1}$ is obtained on $[0,1]$ which is a solution of problem (32).

\section{Conclusion}

We have studied a nonlocal hybrid boundary value problem which contains both left Caputo and right Riemann-Liouville fractional derivatives and integrals and nonlocal hybrid conditions. An existence result is proved by applying a new generalization of Darbo's fixed point theorem associated with measures of noncompactness. The result obtained in this paper is new and significantly contributes to the existing literature on the topic.

\section{Data Availability}

No data were used to support this study.

\section{Conflicts of Interest}

The authors declare that there are no conflicts of interest regarding the publication of this paper.

\section{Acknowledgments}

This research was funded by King Mongkut's University of Technology North Bangkok (contract no. KMUTNB-62KNOW-28). 


\section{References}

[1] I. Podlubny, Fractional Differential Equations, Academic Press, San Diego, CA, USA, 1999.

[2] A. A. Kilbas, H. M. Srivastava, and J. J. Trujillo, Theory and Applications of Fractional Differential Equations, North-Holland Mathematics Studies, Vol. 204, Elsevier, Amesterdam, The Netherlands, 2006.

[3] B. Ahmad, A. Alsaedi, S. K. Ntouyas, and J. Tariboon, Hadamard-Type Fractional Differential Equations, Inclusions and Inequalities, Springer, Cham, Switzerland, 2017.

[4] A. Ergun, "Integral representation for solution of discontinuous diffusion operator with jump conditions," Cumhuriyet Science Journal, vol. 39, no. 1, pp. 842-863, 2018.

[5] A. Ergun and R. Amirov, "Direct and inverse problems for diffusion operator with discontinuity points," TWMS Journal of Pure and Applied Mathematics, vol. 9, no. 1, pp. 9-21, 2019.

[6] R. Amirov, A. Ergun, and S. Durak, "Half-inverse problems for the quadratic pencil of the Sturm-Liouville equations with impulse," Numerical Methods for Partial Differential Equations, vol. 37, no. 1, pp. 915-924, 2021.

[7] B. C. Dhage and V. Lakshmikantham, "Basic results on hybrid differential equations," Nonlinear Analysis: Hybrid Systems, vol. 4, no. 3, pp. 414-424, 2010.

[8] K. Hilal and A. Kajouni, "Boundary value problems for hybrid differential equations with fractional order," Advances in Differential Equations, vol. 2015, p. 183, 2015.

[9] E. T. Karimov, B. López, and K. Sadarangani, "About the existence of solutions for a hybrid nonlinear generalized fractional pantograph equation," Fractional Differential Calculus, vol. 6, no. 1, pp. 95-110, 2016.

[10] N. Mahmudov and M. Matar, "Existence of mild solutions for hybrid differential equations with arbitrary fractional order," TWMS Journal of Pure and Applied Mathematics, vol. 8, pp. 160-169, 2017.

[11] S. Sitho, S. K. Ntouyas, and J. Tariboon, "Existence results for hybrid fractional integro-differential equations, Bound," Value Problems, vol. 2015, p. 113, 2015.

[12] S. Sun, Y. Zhao, Z. Han, and Y. Li, "The existence of solutions for boundary value problem of fractional hybrid differential equations," Communications in Nonlinear Science and Numerical Simulation, vol. 17, no. 12, pp. 4961-4967, 2012.

[13] B. Ahmad, A. Broom, A. Alsaedi, and S. K. Ntouyas, "Nonlinear integro-differential equations involving mixed right and left fractional derivatives and integrals with nonlocal boundary data," Mathematics, vol. 8, no. 3, pp. 1-13, 2020.

[14] O. P. Agrawal, "Formulation of Euler-Lagrange equations for fractional variational problems," Journal of Mathematical Analysis and Applications, vol. 272, no. 1, pp. 368-379, 2002.

[15] L. Zhang, B. Ahmad, and G. Wang, "The existence of an extremal solution to a nonlinear system with the right-handed Riemann-Liouville fractional derivative," Applied Mathematics Letters, vol. 31, pp. 1-6, 2014.

[16] R. Khaldi and A. Guezane-Lakoud, "Higher order fractional boundary value problems for mixed type derivatives," Journal of Nonlinear Functions and Analysis, vol. 30, p. 9, 2017.

[17] A. G. Lakoud, R. Khaldi, and A. Kilicman, "Existence of solutions for a mixed fractional boundary value problem," Advances in Difference Equations, vol. 2017, p. 164, 2017.

[18] A. G. Lakoud, R. Khaldi, and D. F. M. Torres, "On a fractional oscillator equation with natural boundary conditions," Progress in Fractional Differentiation and Applications, vol. 3, no. 3, pp. 191-197, 2017.
[19] S. K. Ntouyas and H. H. Al-Sulami, "A study of coupled systems of mixed order fractional differential equations and inclusions with coupled integral fractional boundary conditions," Advances in Difference Equations, vol. 2020, p. 73, 2020.

[20] B. Ahmad, S. K. Ntouyas, and A. Alsaedi, "Existence theory for nonlocal boundary value problems involving mixed fractional derivatives," Nonlinear Analysis: Modelling and Control, vol. 24, pp. 937-957, 2019.

[21] M. Bouaouid, M. Hannabou, and K. Hilal, "Nonlocal conformable-fractional differential equations with a measure of noncompactness in Banach spaces," Journal of Mathematics, vol. 2020, Article ID 5615080, , 2020.

[22] M. A. Ragusa and A. Scapellato, "Mixed Morrey spaces and their applications to partial differential equations," Nonlinear Analysis: Theory, Methods \& Applications, vol. 151, pp. 51-65, 2017.

[23] M. A. Ragusa and A. Tachikawa, "Regularity for minimizers for functionals of double phase with variable exponents," Advances in Nonlinear Analysis, vol. 9, pp. 710-728, 2020.

[24] E. Set, A. O. Akdemir, and I. Mumcu, "Chebyshev type inequalities for conformable fractional integrals," Miskolc Mathematical Notes, vol. 20, no. 2, pp. 1227-1236, 2019.

[25] M. A. Dokuyucu, E. Celik, H. Bulut, and H. M. Baskonus, "Cancer treatment model with the Caputo-Fabrizio fractional derivative," European Physical Journal-Plus, vol. 133, p. 92, 2018.

[26] I. Koca, E. Akcetin, and P. Yaprakdal, "Numerical approximation for the spread of SIQR model with Caputo fractional order derivative," Turkish Journal of Science, vol. 5, no. 2, pp. 124-139, 2020.

[27] M. A. Dokuyucu and H. Dutta, "A fractional order model for Ebola Virus with the new Caputo fractional derivative without singular kernel," Chaos, Solitons \& Fractals, vol. 134, Article ID 109717, 2020.

[28] M. A. Dokuyucu, "Caputo and Atangana-Baleanu-Caputo fractional derivative applied to Garden equation," Turkish Journal of Science, vol. 5, no. 1, pp. 1-7, 2020.

[29] P. Agarwal and A. A. El-Sayed, "Vieta-Lucas polynomials for solving a fractional-order mathematical physics model," Advances in Differential Equations, vol. 2020, p. 626, 2020.

[30] N. A. Shah, P. Agarwal, J. D. Chung, E. R. El-Zahar, and Y. S. Hamed, "Analysis of optical solitons for nonlinear Schrödinger equation with detuning term by iterative transform method," Symmetry, vol. 12, no. 11, p. 1850, 2020.

[31] P. Agarwal, M. Akbar, R. Nawaz, and M. Jleli, "Solutions of system of Volterra intefro-differential equations using optimal homotopy asymptotic method," Mathematical Methods in the Applied Sciences, vol. 44, no. 3, pp. 1-11, 2020.

[32] J. Banas and K. Goebel, "Measure of noncompactness in Banach spaces," in Lecture Notes in Pure and Applied MathematicsVol. 60, Dekker, New York, NY, USA, 1980.

[33] A. Samadi and M. B. Ghaemi, "An extension of Darbo fixed point theorem and its applications to coupled fixed point and integral equations," Filomat, vol. 28, no. 4, pp. 879-886, 2014.

[34] A. Samadi, "Applications of measure of noncompactness to coupled fixed points and systems of integral equations," Miskolc Mathematical Notes, vol. 19, no. 1, pp. 537-553, 2018. 